\title{
The relation of platelet-lymphocyte ratio and coronary collateral circulation in patients with non-ST segment elevation myocardial infarction
}

\author{
Serkan Akdag, Aytac Akyol, Muntecep Asker, Fatih Ozturk, Hasan Ali Gumrukcuoglu \\ Department of Cardiology, Faculty of Medicine, Yuzuncu Yil University, Van, Turkey
}

Adv Interv Cardiol 2016; 12, 3 (45): 224-230

DOI: 10.5114 /aic.2016.61644

\begin{abstract}
A bstract
Introduction: Recently, platelet-to-lymphocyte ratio (PLR) has emerged as a significant inflammatory marker and a novel predictor of major adverse consequences in cardiovascular disease.

Aim: In this study, we aimed to explore the relationship between PLR and coronary collateral circulation (CCC) in patients with non-ST elevation myocardial infarction (NSTEMI).

Material and methods: Clinical and laboratory data of 386 patients who underwent coronary angiography were evaluated retrospectively. The patients were classified into 2 groups as follows: poor CCC (group 1: Rentrop grades 0-1) and good CCC (group 2: Rentrop grades 2-3). The PLR was calculated from the complete blood count.

Results: The PLR values of the patients with poor CCC were significantly higher than those of patients with good CCC (153.9 \pm 26.6 vs. $129.8 \pm 23.5, p<0.001$ ). In the multiple logistic regression tests, PLR (odds ratio: $1.51,95 \%$ confidence interval: $1.27-1.74$; $p<0.001$ ) and hs-CRP (odds ratio: 1.56, 95\% Cl: 1.03-2.11; $p<0.001$ ) were found to be independent predictors of poor CCC. The receiver operating characteristic (ROC) curve analysis yielded a cutoff value of 140.5 for PLR to predict poor CCC with $79 \%$ sensitivity and $71 \%$ specificity, with the area under the ROC curve being 0.792 (95\% Cl: $0.721-0.864)$.

Conclusions: Our study revealed that high PLR is independently associated with poor coronary collateral circulation in patients with NSTEMI.
\end{abstract}

Key words: angiography, coronary artery disease, cardiology.

\section{Introduction}

Coronary collateral circulation (CCC) is an alternative source of blood supply to an ischemic myocardial region in coronary artery disease (CAD). The development of CCC is one of the important protective adaptations of the heart against ischemia [1]. Presence of poor or good CCC is associated with infarct size, ventricular functions, and survival [2]. Well-developed collaterals diminish the extent of the ischemic area, reduce ventricular aneurysm formation, and improve ventricular functions [3-5].

Platelet activation plays a central role in the initiation and progression of atherosclerosis [6]. Previous studies have demonstrated that increased platelet activation is associated with major adverse cardiovascular consequences [7-9]. On the other hand, a low blood lymphocyte count has been shown to be related to worse cardiovascular outcomes in patients with coronary artery disease.

\section{Aim}

Platelet-to-lymphocyte ratio (PLR) has recently emerged as a significant inflammatory marker and a novel predictor of major adverse consequences in cardiovascular disease [10-12]. Therefore, the aim of this study was to evaluate the relationship between PLR and CCC in patients with non-ST elevation myocardial infarction (NSTEMI).

\section{Material and methods}

\section{Study population}

The study complies with the Declaration of Helsinki, and the trial protocol was approved by the local ethics committee. The retrospective study included 512 patients with NSTEMI who underwent coronary angiography in our catheterization laboratory between May 2012 and January 2015. NSTEMI was defined by the presence of ischemic chest pain, a troponin-l level of $>0.01 \mathrm{ng} /$

\section{Corresponding author:}

Serkan Akdag MD, PhD, Department of Cardiology, Faculty of Medicine, Yuzuncu Yil University, 65000 Van, Turkey, phone: +90 5546983126, e-mail: sercardiocon@hotmail.com

Received: 31.05.2015, accepted: 30.06.2015. 
$\mathrm{ml}$, and notable absence of ST segment elevation on 12-lead electrocardiography (ECG). The exclusion criteria for the present study were as follows: coronary angiography showing a lesion of $<80 \%$ stenosis $(n=71)$, history of percutaneous coronary intervention $(n=19)$ or coronary artery bypass grafting $(n=9)$, active and ongoing infection $(n=14)$, moderate to severe renal or hepatic diseases $(n=6)$, hemodynamically significant ventricular arrhythmias $(n=4)$, and malignancies $(n=3)$. After applying the exclusion criteria, 386 patients with NSTEMI were enrolled in the study.

Risk factors and characteristic features including age, gender, status of smoking, hypertension, diabetes mellitus (DM), hyperlipidemia, and family history were recorded. Also, hematological parameters, serum creatinine, blood glucose, troponin, creatine kinase-myocardial band (CKMB), lipid profile, high-sensitivity C-reactive protein (hs-CRP), blood pressure, heart rate, and history of drug use were evaluated for each patient. Prior to the angiography, transthoracic echocardiography was performed for each patient and left ventricular ejection fraction (LVEF) was assessed using the biplane Simpson method. Hematological parameters including hemoglobin level, white blood cells, platelet counts, lymphocyte counts, and mean platelet volume were measured to achieve the automated complete blood count (CBC) using a Coulter LH 780 Hematology Analyzer (Architect plus ci16200 Abbott Illinois USA). Hypertension was defined as systolic blood pressure of $140 \mathrm{~mm} \mathrm{Hg}$ or greater and/ or diastolic pressure of $90 \mathrm{~mm} \mathrm{Hg}$ or greater measured at least 2 times, or the use of antihypertensive medications. Diabetes mellitus was defined based on a history of antidiabetic medication or a fasting glucose level of $126 \mathrm{mg} / \mathrm{dl}$ or greater.

\section{Coronary angiography}

The Judkins technique was used to perform the quantitative coronary angiography via the femoral artery. To measure the coronary artery lesion and CCC, the data obtained for each patient were evaluated by two physicians blinded to the clinical and laboratory outcomes. The evaluations included the angiographies that had an epicardial coronary stenosis of $80 \%$ or greater. The Rentrop classification was used for grading CCC [13]. In this classification, grade 0 implies scarcity of filling in the supplementary vessels, grade 1 implies filling in side branches via collateral channels with no visibility of epicardial artery, grade 2 implies incomplete filling in the major epicardial coronary artery through collateral channels, and grade 3 implies complete filling in the major epicardial coronary artery. The patients were divided into two groups: poor CCC (group 1: grades 0-1) and good CCC (group 2: grades 2-3). Multivessel coronary artery disease was defined as the detection of a lesion in 2 or more major epicardial arteries. Additionally, the Gensini scoring system was used to identify the severity of CAD. In this system, 1 point indicates $1 \%$ to $25 \%$ stenosis, 2 points indicate $26 \%$ to $50 \%, 4$ points indicate $51 \%$ to $75 \%, 8$ points indicate $76 \%$ to $90 \%$, 16 points indicate $91 \%$ to $99 \%$ stenosis, and 32 points indicate total occlusion.

\section{Statistical analysis}

All the data were analyzed using SPSS 17.0 for Windows (SPSS Inc., USA). Continuous variables are shown as mean \pm standard deviation and categorical variables are shown as percentages. The Kolmogorov-Smirnov test was used to confirm the normality of distribution for continuous variables. Student's $t$-test, the Mann-Whitney $U$ test or the $\chi^{2}$ test was used for comparisons between groups, as necessary. The grades were compared using the analysis of variance and Tukey's honest significant difference test as the post hoc test. Independent markers of CCC were identified using multiple logistic regression analysis with the variables showing marginal associations on univariate testing $(p<0.10)$. The cut-off value of PLR in the prediction of CCC was identified using receiver operating characteristic (ROC) analysis. To analyze the correlation between the variables, Pearson or Spearman correlation was used. The $p$ values less than 0.05 were accepted as statistically significant.

\section{Results}

A total of 386 patients with NSTEMI were included in the study. Of these, 185 patients (mean age: $59.3 \pm 11.3$ years, $62.2 \%$ male) were in the poor CCC group, and 201 patients (mean age: $57.8 \pm 10.4$ years, $58.7 \%$ male) were in the good CCC group. Comparisons of the baseline clinical, laboratory, and angiographic characteristics of the patients according to the coronary collateral formation are summarized in Tables I and II. No significant differences were found in the cardiovascular risk profiles of both groups such as presence of hypertension, DM, family history of coronary artery disease, smoking status, hyperlipidemia, and systolic and diastolic blood pressure. Sex, age, LVEF, previous medications and the number of diseased vessels were similar across the two groups. Additionally, no significant difference was found in the Gensini scores in two groups ( $62.6 \pm 27.1$ vs. $61.4 \pm 26.8, p=0.568)$

Patients with poor CCC (Rentrop grades 0 and 1) had a significantly higher platelet count, mean platelet volume and hs-CRP as well as a lower value of absolute lymphocyte count compared to patients with good CCC (Rentrop grades 2 and 3) (Table I). Compared to the patients with good CCC, patients with poor CCC manifested significantly higher PLR values ( $153.9 \pm 26.6$ vs. $129.8 \pm 23.5$, $p<0.001)$. An inverse stepwise association was present between collateral score and PLR values (Figure 1). The PLR values decreased with increasing Rentrop grade, with a mean value of $162.3 \pm 30.6,151.2 \pm 27.4,133.5$ $\pm 21.7,123.6 \pm 24.6$ in patients with Rentrop grades of 0 , 
Table I. Baseline characteristics according to coronary collateral circulation

\begin{tabular}{|c|c|c|c|}
\hline Parameter & $\begin{array}{l}\text { Poor CCC } \\
(n=185) \\
\end{array}$ & $\begin{array}{l}\text { Good CCC } \\
(n=201)\end{array}$ & $P$-value \\
\hline Sex, male (\%) & 62.2 & 58.7 & 0.489 \\
\hline Age [years] & $59.3 \pm 11.3$ & $57.8 \pm 10.4$ & 0.168 \\
\hline Duration of chest pain [h] & $7.7 \pm 6.1$ & $7.5 \pm 5.9$ & 0.664 \\
\hline Hypertension (\%) & 56 & 51 & 0.380 \\
\hline Diabetes (\%) & 21 & 17 & 0.362 \\
\hline Smoking (\%) & 41 & 36 & 0.335 \\
\hline Family history of CAD (\%) & 19 & 17 & 0.605 \\
\hline Total cholesterol [mg/dl] & $191.3 \pm 97.3$ & $183.6 \pm 88.6$ & 0.264 \\
\hline Triglyceride [mg/dl] & $172.7 \pm 112.5$ & $165.9 \pm 106.8$ & 0.433 \\
\hline LDL cholesterol [mg/dl] & $119.4 \pm 53.2$ & $113.9 \pm 49.5$ & 0.378 \\
\hline HDL cholesterol [mg/dl] & $37.3 \pm 6.3$ & $36.5 \pm 5.8$ & 0.248 \\
\hline Fasting glucose [mg/dl] & $129.4 \pm 51.4$ & $122.6 \pm 49.1$ & 0.048 \\
\hline Creatinine $[\mathrm{mg} / \mathrm{dl}]$ & $0.93 \pm 0.23$ & $0.95 \pm 0.27$ & 0.131 \\
\hline Baseline troponin [ $\mathrm{\mu g} / \mathrm{l}]$ & $2.8 \pm 3.4$ & $3.2 \pm 3.9$ & 0.167 \\
\hline Baseline CK-MB [IU/I] & $28.2 \pm 15.8$ & $30.7 \pm 17.9$ & 0.141 \\
\hline Platelet count $\left[\times 10^{3} \mu / l\right]$ & $277.9 \pm 43.1$ & $260.8 \pm 39.3$ & $<0.01$ \\
\hline Lymphocyte $\left[\times 10^{3} \mathrm{\mu} / \mathrm{l}\right]$ & $1.8 \pm 0.5$ & $2.0 \pm 0.6$ & $<0.01$ \\
\hline Platelet/lymphocyte ratio & $153.9 \pm 26.6$ & $129.8 \pm 23.5$ & $<0.01$ \\
\hline WBC $\left[\times 10^{3} \mu / l\right]$ & $8.3 \pm 2.4$ & $7.9 \pm 2.3$ & 0.019 \\
\hline Mean platelet volume [fl] & $8.8 \pm 1.1$ & $8.3 \pm 0.9$ & $<0.01$ \\
\hline $\mathrm{Hs}-\mathrm{CRP}[\mathrm{mg} / \mathrm{l}]$ & $3.2 \pm 0.9$ & $2.7 \pm 0.8$ & $<0.01$ \\
\hline Heart rate [beats/min] & $76.4 \pm 11.3$ & $78.3 \pm 10.6$ & 0.118 \\
\hline Systolic BP [mm Hg] & $131.1 \pm 25.7$ & $129.3 \pm 21.8$ & 0.143 \\
\hline Diastolic BP [mm Hg] & $76.4 \pm 15.3$ & $74.8 \pm 14.1$ & 0.108 \\
\hline LVEF (\%) & $51.2 \pm 8.2$ & $49.9 \pm 7.8$ & 0.119 \\
\hline \multicolumn{4}{|l|}{ Previous medications (\%): } \\
\hline Aspirin & 16 & 15 & 0.831 \\
\hline ACE inhibitors/ARBs & 32 & 35 & 0.542 \\
\hline Calcium channel blockers & 16 & 13 & 0.533 \\
\hline Diuretics & 12 & 14 & 0.665 \\
\hline$\beta$-blockers & 23 & 20 & 0.501 \\
\hline Statin & 19 & 17 & 0.609 \\
\hline Insulin & 6 & 7 & 0.852 \\
\hline Oral antidiabetics & 17 & 14 & 0.363 \\
\hline
\end{tabular}

$A C E$ - angiotensin-converting enzyme, $A R B$ - angiotensin receptor blocker, CAD - coronary artery disease, HDL - high-density lipoprotein, hs-CRP - high-sensitivity C-reactive protein, LDL - low-density lipoprotein, LVEF - left ventricular ejection fraction, WBC - white blood cell count. Bold values indicate univariate variables entered into multivariate model, $p<0.01$. 
Table II. Coronary angiographic findings of the study population

\begin{tabular}{|c|c|c|c|}
\hline Parameter & $\begin{array}{l}\text { Poor CCC } \\
(n=185) \\
\end{array}$ & $\begin{array}{l}\text { Good CCC } \\
(n=201) \\
\end{array}$ & $P$-value \\
\hline 1-vessel disease: & $94(51 \%)$ & $91(45 \%)$ & 0.278 \\
\hline LAD stenosis & $43(23 \%)$ & $39(19 \%)$ & 0.358 \\
\hline LCX stenosis & 31 (17\%) & $33(16 \%)$ & 0.929 \\
\hline RCA stenosis & 20 (11\%) & $19(10 \%)$ & 0.660 \\
\hline 2-vessel disease: & $59(32 \%)$ & $68(34 \%)$ & 0.686 \\
\hline LAD and LCX stenosis & $35(19 \%)$ & $33(16 \%)$ & 0.521 \\
\hline LAD and RCA stenosis & $10(5 \%)$ & $16(8 \%)$ & 0.318 \\
\hline LCX and RCA stenosis & $14(8 \%)$ & $19(10 \%)$ & 0.509 \\
\hline 3-vessel disease & 32 (17\%) & $42(21 \%)$ & 0.371 \\
\hline Number of diseased coronary vessels & $1.7 \pm 0.6$ & $1.8 \pm 0.8$ & 0.244 \\
\hline \multicolumn{4}{|l|}{ Rentrop collateral grades: } \\
\hline 0 & $46(12 \%)$ & - & - \\
\hline 1 & $139(36 \%)$ & - & - \\
\hline 2 & - & $123(32 \%)$ & - \\
\hline 3 & - & $78(20 \%)$ & - \\
\hline Gensini scores & $61.4 \pm 26.8$ & $62.6 \pm 27.1$ & 0.568 \\
\hline
\end{tabular}

CCC - coronary collateral circulation, $L A D$ - left anterior descending coronary artery, $L C X$ - left circumflex coronary artery, RCA - right coronary artery.

$1,2$, and 3 , respectively ( $p$ value for trend $<0.001)$. Additionally, there was a positive correlation between Gensini score and PLR $(r=0.420, p<0.001)$.

The ROC curve analysis was performed to detect the best cutoff value of PLR in the prediction of poor CCC. The ROC analysis yielded a cutoff value of 140.5 for PLR

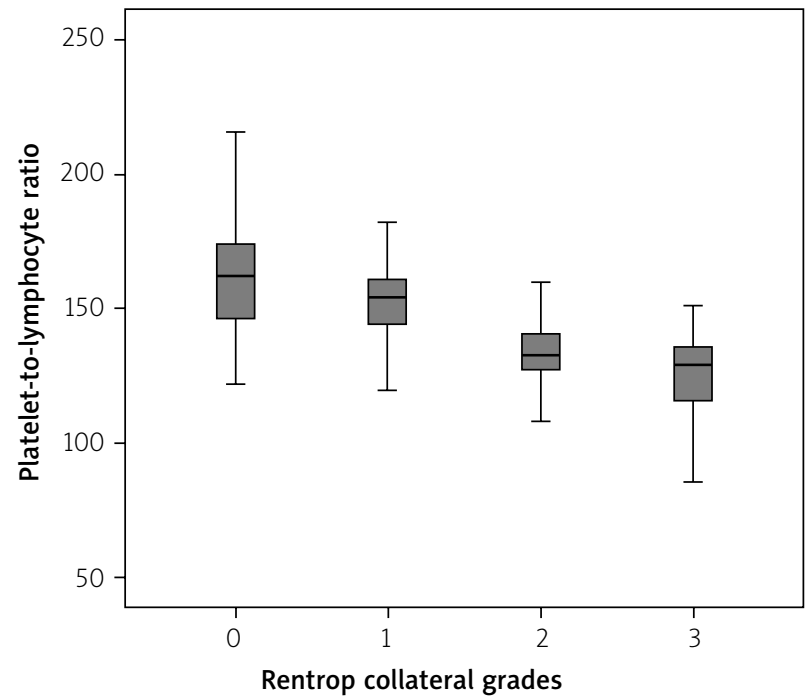

Figure 1. Platelet/lymphocyte ratio according to Rentrop grades to predict insufficient CCC with $79 \%$ sensitivity and $71 \%$ specificity, with the area under the ROC curve being 0.792 (95\% Cl: 0.721-0.864, Figure 2).

Multiple regression tests (univariate and multivariate logistic regression tests) were employed to determine the independent predictors of poor CCC. The variables

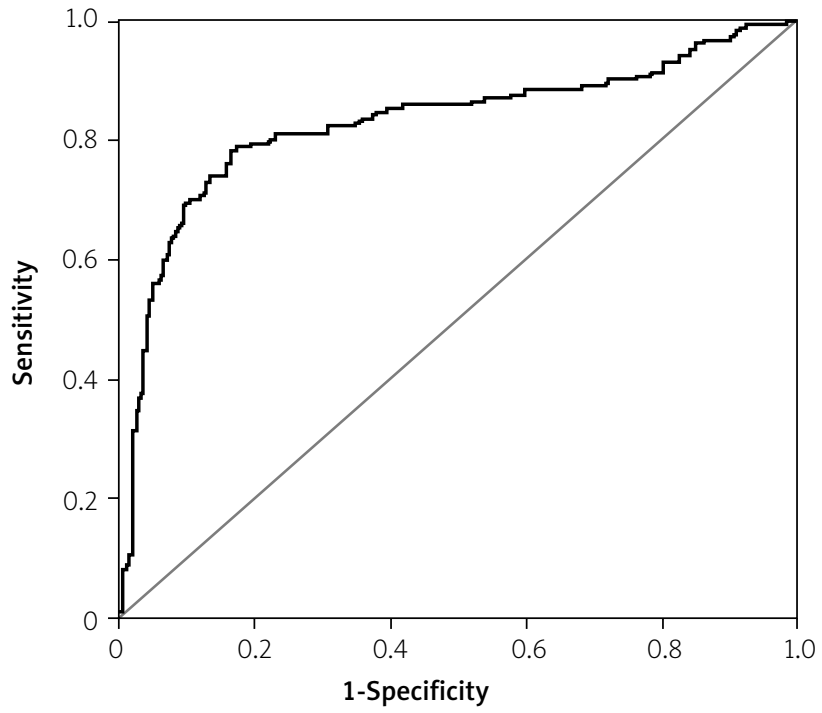

Figure 2. Receiver operating characteristic (ROC) curve for platelet-to-lymphocyte ratio in prediction of poor coronary collateral circulation 
Table III. Univariate and multivariate logistic regression analysis to detect independent predictors of poor coronary collateral circulation

\begin{tabular}{lcccc} 
& \multicolumn{2}{c}{ Univariate } & \multicolumn{2}{c}{ Multivariate } \\
\cline { 2 - 5 } & OR $(95 \% \mathrm{Cl})$ & $P$-value & OR $(95 \% \mathrm{Cl})$ & $P$-value \\
\hline Age & $0.99(0.96-1.03)$ & 0.66 & $1.08(0.95-1.25)$ & 0.27 \\
\hline Sex & $0.71(0.56-1.27)$ & 0.24 & $0.91(0.13-4.28)$ & 0.68 \\
\hline Smoking & $0.48(0.21-1.14)$ & 0.18 & $0.82(0.15-3.69)$ & 0.49 \\
\hline Fasting glucose & $0.98(0.97-0.99)$ & 0.63 & - & - \\
\hline PLR & $1.47(1.25-1.66)$ & $<0.001$ & - & - \\
\hline WBC & $0.87(0.73-1.04)$ & 0.23 & -001 \\
\hline MPV & $1.48(1.08-1.86)$ & $<0.001$ & $1.27-1.74)$ & - \\
\hline hs-CRP & $1.64(1.28-2.02)$ & $<0.001$ & - & $<0.001$
\end{tabular}

$O R$ - odds ratio, $C l$ - confidence interval, hs-CRP - high-sensitivity C-reactive protein, $P L R$ - platelet/lymphocyte ratio, MPV - mean platelet volume, WBC - white blood cell.

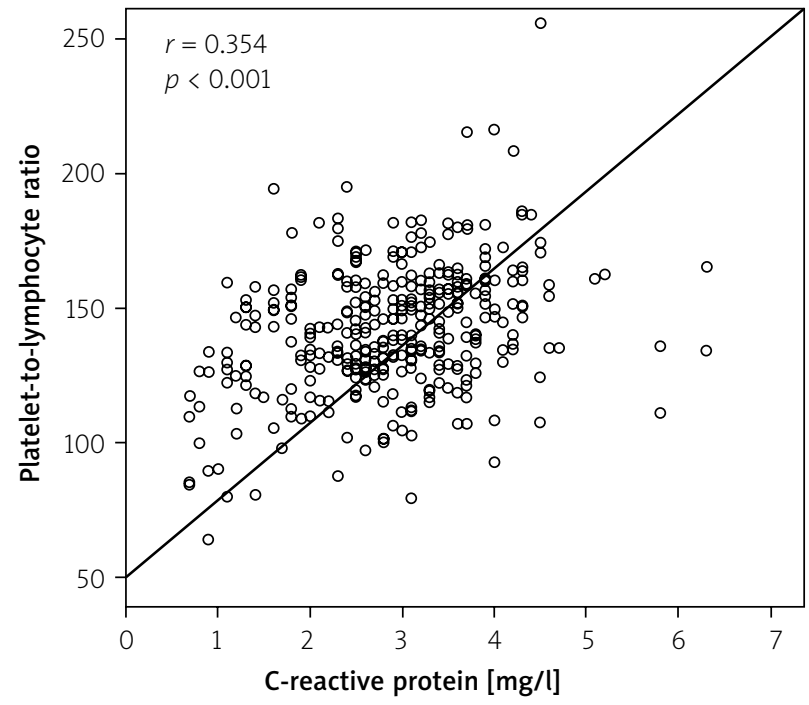

Figure 3. Scatter plot showing positive correlation between platelet-to-lymphocyte ratio (PLR) and serum high-sensitivity C-reactive protein (hs-CRP) levels

platelet count, lymphocyte count, mean platelet volume, hs-CRP and PLR were found to have significance in the univariate analysis. Furthermore, PLR (unit $=10$; odds ratio (OR) 1.51, 95\% confidence interval (CI): 1.27-1.74; $p<0.001)$ and hs-CRP (OR $=1.56,95 \% \mathrm{Cl}: 1.03-2.11$; $p<0.001$ ) were found to be independent predictors of poor CCC in multivariate logistic regression analysis (Table III). Moreover, PLR showed a positive correlation with hs-CRP $(r=0.354, p<0.001)$ (Figure 3$)$.

\section{Discussion}

In this study, PLR value, platelet count, and serum hsCRP level were higher in NSTEMI patients with poor CCC compared to those with good CCC. Moreover, a negative correlation was detected between PLR and collateral score. The results also suggested that PLR is associated with poor CCC and a high PLR is a significant predictor of poor CCC development in patients with NSTEMI.

The collateral coronary arteries in an individual without CAD may not be visible on angiography; however, they become angiographically visible if a severe narrowing or complete obstruction occurs in the coronary arteries [14, 15]. Development of CCC is triggered by the formation of new vessels (angiogenesis) and/or the growth of preexisting arterioles (arteriogenesis) [16]. Also, development of CCC can be affected by a number of factors including the severity and rate of progression of stenosis, hypertension, DM, oxidative stress, endothelial injury, endogenous mediators and physical exercise, status of smoking, higher red cell distribution width, and certain drugs $[16,17]$. Development of good CCC in patients with acute coronary syndrome has been shown to be associated with good myocardial perfusion, improved recovery of ventricular functions, and reduced risk of major long-term cardiovascular consequences [18-20].

Atherosclerosis is a major cause of cardiovascular diseases, which are one of the leading causes of mortality worldwide [21]. The initiation and proliferation of atherosclerosis in the coronary arteries can be associated with numerous factors. Among these, inflammation has a substantial role in the development of atherosclerosis. In cases of chronic inflammation, increased lymphocyte apoptosis leads to a decreased lymphocyte count. In cases of stress, the leukocyte production in bone marrow leads to a decreased lymphocyte count and an increase in the neutrophil count. Although neutrophils lead to a harmful inflammatory reaction, lymphocytes represent a more appropriate immune response [22]. Prior studies 
have shown that decreased lymphocyte count is a useful diagnostic and prognostic marker in patients with acute coronary syndrome and it is associated with a bad prognosis in these patients. Ommen et al. reported that decreased lymphocyte count was significantly associated with survival in patients with stable coronary artery disease and it had a potential independent prognostic value for these patients [23].

Recent studies have demonstrated that increased platelet count and decreased lymphocyte count are associated with bad prognosis in patients with coronary artery diseases $[24,25]$. Yuksel et al. demonstrated that higher PLR is associated with the severity of CAD [26]. Akboga et al. reported the association between higher PLR and severity of coronary atherosclerosis in patients with stable CAD [27]. In our study, there was a positive correlation between PLR and severity of CAD.

Azab et al. reported that increased PLR was an independent predictor of long-term mortality in NSTEMI patients [28]. Akkaya et al. found that the PLR was associated with an increase in adverse clinical conditions and mortality within the 6 months after percutaneous coronary intervention ( $\mathrm{PCl}$ ) in STEMI patients [29]. Gary et al. showed that PLR established a significant correlation with inflammatory indices such as C-reactive protein and fibrinogen in patients with limb ischemia [30]. In our study, we also found a positive correlation between PLR and hs-CRP.

Platelets involve numerous angiogenesis promoters and inhibitors, and thus they regulate the formation of new blood vessels during wound healing, tumor growth, and while responding to ischemia [31]. These angiogenesis promoters include vascular endothelial growth factor, platelet-derived growth factor, basic fibroblastic growth factor, epidermal growth factor, and matrix metalloproteinases [32]. On the other hand, the angiogenesis inhibitors are also available in platelets. Of these inhibitors, angiostatin is one of the most important ones, which has a critical role in coronary collateral formation. Recent studies have shown that angiostatin is an important mediator in the reduction of coronary angiogenesis during the inhibition of nitric oxide synthesis and also that the angiostatin levels in pericardial fluid show a negative correlation with the collateral formation in patients treated with coronary artery bypass surgery [32]. However, CAD is usually associated with thrombocytosis, which has been commonly reported as a poor prognostic indicator [25]. Moreover, increased platelet counts have been shown to be associated with increased risk of thrombotic problems, and both atherogenesis and its complications result from platelets [33]. For this reason, an elevation in platelet counts may be effective both in the development and the outcomes of CAD. Acar et al. reported that higher PLR values are closely associated with poorer CCC in stable angina patients with chronic total occlusions [34].
Şahin et al. demonstrated that poor CCC is associated with increased platelet counts in patients with stable CAD [35]. Similarly, we also found that poor CCC showed a positive correlation with PLR.

Development and functions of coronary collateral arteries have been subject to a long-term debate. It has been reported that there is a positive correlation between the severity of obstruction in the coronary arteries and the degree of coronary collateral formation [36]. However, Acar et al. reported that there is no significant relationship between the severity of coronary artery disease and CCC in patients with chronic total occlusion [34]. In our study, no significant correlation was found between the Gensini scores and collateral circulation. The degree of development of CCC may be different in individuals with the same severity of coronary artery stenosis. Moreover, development of CCC may be affected by numerous endogenous mediators including endothelial dysfunction associated with neurohumoral markers, inflammatory indices, nitric oxide (NO), and vascular endothelial growth factor (VEGF) and by the cytokines released from inflammatory cells and proinflammatory enzymes [16]. The PLR is calculated from white blood cell count (WBC). In recent studies, increased PLR has been reported as a new independent predictor of long-term survival in patients with acute coronary syndrome. However, the association between the CCC development and PLR in NSTEMI patients has not been reported. In this study, we found that increased PLR is an independent predictor of poor CCC.

\section{Conclusions}

Our study results demonstrated that high PLR was associated with poor CCC in patients with NSTEMI. This study is the first report to demonstrate the association between CCC development and PLR in NSTEMI patients. PLR can be used as a practical, inexpensive, and important tool for predicting the degree of coronary collateral formation in patients with NSTEMI. Further studies are needed to investigate the cause-and-effect relationship between PLR and CCC.

\section{Conflict of interest}

The authors declare no conflict of interest.

\section{References}

1. Celik T, Celik M, Iyisoy A. Coronary collateral circulation. Turk Kardiyol Dern Ars 2010; 38: 505-14.

2. Imhof BA, Aurrand-Lions M. Angiogenesis and inflammation face off. Nat Med 2006; 12: 171-2.

3. Hirai T, Fujita M, Nakajima H, et al. Importance of collateral circulation for prevention of left ventricular aneurysm formation in acute myocardial infarction. Circulation 1989; 79: 791-6.

4. Nohara R, Kambara H, Murakami T, et al. Collateral function in early acute myocardial infarction. Am J Cardiol 1983; 52: 955-9. 
5. Saito $Y$, Yasuno $M$, Ishida $M$, et al. Importance of coronary collaterals for restoration of left ventricular function after intracoronary thrombolysis. Am J Cardiol 1985; 55: 1259-63.

6. Pekdemir H, Polat G, Cin VG, et al. Elevated plasma endothelin-1 levels in coronary sinus during rapid right atrial pacing in patients with slow coronary flow. Int J Cardiol 2004; 97: 35-41.

7. Thaulow E, Erikssen J, Sandvik L, et al. Blood platelet count and function are related to total and cardiovascular death in apparently healthy men. Circulation 1991; 84: 613-7.

8. lijima R, Ndrepepa G, Mehilli J, et al. Relationship between platelet count and 30 day clinical outcomes after percutaneous coronary interventions. Pooled analysis of four ISAR trials. Thromb Haemost 2007; 98: 852-7.

9. Vidwan P, Lee S, Rossi JS, et al. Relation of platelet count to bleeding and vascular complications in patients undergoing coronary angiography. Am J Cardiol 2010; 105: 1219-22.

10. Proctor MJ, Morrison DS, Talwar D, et al. A comparison of inflammation-based prognostic scores in patients with cancer. A Glasgow Inflammation Outcome Study. Eur J Cancer 2011; 47: 2633-41.

11. Smith RA, Bosonnet L, Raraty M, et al. Preoperative platelet-lymphocyte ratio is an independent significant prognostic marker in resected pancreatic ductal adenocarcinoma. Am J Surg 2009; 197: 466-72.

12. Smith RA, Ghaneh P, Sutton R, et al. Prognosis of resected ampullary adenocarcinoma by preoperative serum CA19-9 levels and platelet-lymphocyte ratio. J Gastrointest Surg 2008; 12: 1422-8.

13. Rentrop KP, Thornton JC, Feit F, et al. Determinants and protective potential of coronary arterial collaterals as assessed by an angioplasty model. Am J Cardiol 1988; 61: 677-84.

14. Levin DC. Pathways and functional significance of the coronary collateral circulation. Circulation 1974; 50: 831-7.

15. Elayda MA, Mathur VS, Hall RJ, et al. Collateral circulation in coronary artery disease. Am J Cardiol 1985; 55: 58-60.

16. Seiler C. The human coronary collateral circulation. Eur J Clin Invest 2010; 40: 465-76.

17. Tanboga IH, Topcu S, Nacar T, et al. Relation of coronary collateral circulation with red cell distribution width in patients with non-ST elevation myocardial infarction. Clin Appl Thromb Hemost 2014; 20: 411-5.

18. McMurtry MS, Lewin AM, Knudtson ML, et al. The clinical profile and outcomes associated with coronary collaterals in patients with coronary artery disease. Can J Cardiol 2011; 27: 581-8.

19. Ilia R, Wolak A, Amit G, et al. Collateral blood flow can predict myocardial blush grade in primary coronary intervention. Catheter Cardiovasc Interv 2012; 80: 67-70.

20. Nathoe HM, Koerselman J, Buskens E, et al. Determinants and prognostic significance of collaterals in patients undergoing coronary revascularization. Am J Cardiol 2006; 98: 31-5.

21. Lopez AD, Mathers CD, Ezzati M, et al. Global and regional burden of disease and risk factors, 2001: systematic analysis of population health data. Lancet 2006; 367: 1747-57.

22. Zouridakis EG, Garcia-Moll X, Kaski JC. Usefulness of the blood lymphocyte count in predicting recurrent instability and death in patients with unstable angina pectoris. Am J Cardiol 2000; 86: 449-51.

23. Ommen SR, Gibbons RJ, Hodge DO, et al. Usefulness of the lymphocyte concentration as a prognostic marker in coronary artery disease. Am J Cardiol 1997; 79: 812-4.

24. Nikolsky E, Grines CL, Cox DA, et al. Impact of baseline platelet count in patients undergoing primary percutaneous coronary intervention in acute myocardial infarction (from the CADILLAC trial). Am J Cardiol 2007; 99: 1055-61.

25. Yilmaz S, Sen F, Unal S, et al. Usefulness of the platelet-to lymphocyte ratio to predict bare metal stent restenosis. Scand Cardiovasc J 2015; 49: 39-44.

26. Yuksel M, Yıldız A, Oylumlu M, et al. The association between platelet/lymphocyte ratio and coronary artery disease severity. Anatol J Cardiol 2015; 15: 640-7.

27. Akboga MK, Canpolat U, Yayla C, et al. Association of platelet to lymphocyte ratio with inflammation and severity of coronary atherosclerosis in patients with stable coronary artery disease. Angiology 2016; 67: 89-95.

28. Azab B, Shah N, Akerman M, et al. Value of platelet/lymphocyte ratio as a predictor of all-cause mortality after non-ST-elevation myocardial infarction. J Thromb Thrombolysis 2012; 34: 326-34.

29. Akkaya E, Gul M, Ugur M. Platelet to lymphocyte ratio: a simple and valuable prognostic marker for acute coronary syndrome. Int J Cardiol 2014; 177: 597-8.

30. Gary T, Pichler M, Belaj K, et al. Platelet-to-lymphocyte ratio: a novel marker for critical limb ischemia in peripheral arterial occlusive disease patients. PLoS One 2013; 8: e67688.

31. Radziwon-Balicka A, Moncada de la Rosa C, Jurasz P. Platelet associated angiogenesis regulating factors: a pharmacological perspective. Can J Physiol Pharmacol 2012; 90: 679-88.

32. Matsunaga T, Chilian WM, March K. Angiostatin is negatively associated with coronary collateral growth in patients with coronary artery disease. Am J Physiol Heart Circ Physiol 2005; 288: 2042-6.

33. Stissing T, Dridi NP, Ostrowski SR, et al. The influence of low platelet count on whole blood aggregometry assessed by Multiplate. Clin Appl Thromb Hemost 2011; 17: 211-7.

34. Acar G, Kalkan ME, Avci A, et al. The relation of platelet-lymphocyte ratio and coronary collateral circulation in patients with stable angina pectoris and chronic total occlusion. Clin Appl Thromb Hemost 2015; 21: 462-8.

35. Şahin I, Karabulut A, Kaya A, et al. Increased level of red cell distribution width is associated with poor coronary collateral circulation in patients with stable coronary artery disease. Turk Kardiyol Dern Ars 2015; 43: 123-30.

36. Pohl T, Seiler C, Billinger $M$, et al. Frequency distribution of collateral flow and factors influencing collateral channel development. functional collateral channel measurement in 450 patients with coronary artery disease. J Am Coll Cardiol 2001; 38: $1872-8$ 